\title{
Roles of gray-scale, Color-Doppler ultrasound and sonoelastography for assessment of thyroid nodules: nodule size correlation
}

\author{
Hamad Elniel Hassan Eltyib ${ }^{1}$, Bushra Hussein Ahmed ${ }^{2}$ and Alsafi A. Abd Alla ${ }^{3}$ \\ ${ }^{1}$ King Abdulaziz University Jeddah, Faculty of Applied Medical Sciences, Department of Diagnostic Radiology, \\ ${ }^{2}$ National Ribat University and ${ }^{3}$ College of Medical Radiologic Science, Sudan University of Science and \\ Technology, Khartoum-Sudan.
}

\begin{abstract}
Objective: To evaluate the predictive value of roles of gray-scale, color-Doppler ultrasound, and sonoelastography for the assessment of thyroid nodule to determine whether nodule size affects the differential diagnosis of benign and malignant, with fine needle aspiration cytology analysis as the reference standard.

Methods: A total of 270 consecutive patients (mean age, 35 55 years; range, 30-50 years; 25males and 245 females) with 300 thyroid nodules were examined by gray-scale, color-Doppler ultrasound, and 100 patients examined by sonoelastography in this prospective study. All patients underwent surgery and the final diagnosis was obtained from fine needle aspiration cytology analysis.

Results: Three hundred nodules (206 benign, 94 malignant) were divided into small (SNs, $\leq 1 \mathrm{~cm}, n=124$ ) and large (LNs, >1Cm, n=178) nodules. Microcalcifications were more frequent in malignant LNs than in malignant SNs, but showed no significant difference between benign LNs and SNs. Poorly-circumscribed margins were not significantly different between malignant $S N s$ and $L N s$, but were less frequent in benign LNs than in benign SNs. Among all nodules, marked intranodular vascularity was more frequent in LNs than in SNs. By comparison, shape ratio of anteroposterior to transverse dimensions $(A / T) \geq 1$ was less frequent in $L N$ s than in SNs. Otherwise, among all nodules, marked hypoechogenicity and elasticity score of 4-5 showed no significant difference between LNs and SNs.

Conclusions: The predictive values of microcalcifications, nodular margins, A/T ratio, and marked intranodular vascularity depend on nodule size, but the predictive values of echogenicity and elastography do not depend on nodule size.
\end{abstract}

Key words: Elastography; Thyroid nodules; Ultrasound. Malignant. Nodule size.

\section{Introduction}

Thyroid nodules are one of the mostly encountered medical problems that need precise diagnosis and specification prior to decision making regarding treatment. They are found in $4 \%-8 \%$ of adults by palpation, $41 \%$ by ultrasonography (US), and $50 \%$ by pathologic postmortem examination. The prevalence of thyroid cancer is as high as $5 \%-10 \%$. [1], and in other literature it is about 5\% 1-3 [2\} Papillary carcinoma is the most common ( $75 \%-80 \%)$. Other types include follicular carcinoma (10\%-20\%), medullar carcinoma $(3 \%-5 \%)$, and an plastic carcinoma (1\%-2\%). Lymphoma and metastases of the thyroid gland are not common. [1\}

Ultrasonography has been used for decades to diagnose thyroid nodules and to select cases for fine needle aspiration biopsy (FNAB) in which malignancy is suspected. However, conventional US are not as useful for differentiating benign and malignant nodules. [3, 4, 5] In addition, the sensitivity, specificity, negative and positive predictive values are considerably variable from study to another. $[6,7,8]$ FNAB provides the best method to differentiate benign from malignant nodules. [9] although it is recommended for all nodules with a diameter more than $1 \mathrm{~cm}$, and in smaller nodules having findings suggestive of malignancy, It is estimated that about 250,000 : 300,000 thyroid FNA biopsies are performed in the United States every year. However, a large percentage (approximately $70 \%$ ) of these biopsies turns out to be benign. [10] In some literatures, $15 \%$ to $30 \%$ of samples obtained by FNAB are classified as indeterminate, and patients are referred for thyroidectomy or lobectomy. Thyroid carcinoma is ultimately confirmed in less than $20 \%$ of these cases leaving unsatisfactory diagnostic workup of thyroid nodules. [11]_In addition, US-guided FNAB is an invasive procedure that has disadvantages e.g. sampling errors, time and money consumption, painful, not easy to be performed for very small nodules and it is associated with minor complications. [12] Palpation is a basic and important method in the assessment of thyroid nodules. Malignant nodules tend to be much harder than benign ones. However, the stiffness of thyroid nodules in palpation is subjective and can't be an accurate indicator for malignancy [13] although there are many criteria that are indicator for malignancy using conventional Ultrasound e.g. nodule echogenicity, microcalcification, blurred or speculated margin, absent halo sign and abnormal vascularity, yet it 
does not provide direct information about the hardness of a nodule. Ultrasound elastography (USE) was developed to obtain information on tissue stiffness noninvasively. It was first applied for characterization of breast lesions, and recently it is applied for prostatic and thyroid tumors. [2] USE is based on the reconstruction of tissue stiffness by measuring the degree of tissue's deformation in response to the application of an external force. [14] The softer parts of tissues deform easier than the harder parts under compression, thus allowing an objective determination of tissue stiffness. [2]This technique has been shown to be useful in the differential diagnosis between benign and malignant tumors. Most malignant tumors are stiff and hard compared to the surrounding normal tissues, while most benign tumors are softer. [14] Many scoring systems were applied to reflect tissue elasticity; the most feasible of them is that of I toh A et al, 2006 who assigned 5 scores depending of the color shown by the tissue when it is subjected to pressure. Tissue stiffness on USE was scored from 1 (greatest elastic strain) to 5 (no strain) based on subjective analysis of the elastogram images and color. This scoring system was used to differentiate benign and malignant lesions as shown in table 1. [15]

The purpose of this study was to define the roles of gray-scale, color-Doppler ultrasound, and sonoelastography for assessment of thyroid nodules to determine if nodule size affects differential diagnosis of benign and malignant using FNAC as the reference standard.

\section{Patients and methods:}

"Patients", This is prospective study after obtaining an ethical approval, 300 patients were included in this study ( 30 males and 270 females) ranging in age from 19 to 75 years; with the mean age are 43 . The study was done at the diagnostic radiology department, KAUH during the 2011- 2013year. All patients provided written consent before they were subjected to grey scale US and Doppler study followed by free hand USE using a real-time scale of 1 to 5 , where 5 corresponds to the highest tissue stiffness. Tissue stiffness on USE was scored into five scores according to Ueno E, 2006. All cases were done using i22 Philips US machine and high frequency $(5-12 \mathrm{MHz})$ linear probe. Images were taken before and after compression. The color scale varied from red (softest tissue) to blue (hardest tissue). Although many patients had multiple nodules, yet we applied USE for the most suspicious ones only.30 patients were excluded from the study either because they refused FNAB or due to insufficient sample from tiny nodules. So, the remaining 270 were only included in the study (145 females and 25 males). FNA biopsy/ cytology were done by an expert radiologist under strict aseptic conditions, using local anesthesia and appropriate needles. Written consent was provided by every patient. All specimens were referred to the central laboratory department at KAUH for interpretation.

"Statistical analysis", Demographic patients data together with USE results and FNAB results were collected and Statistical analyses were performed using the IBM SPSS Statistics version 21.0 (IBM Company, New York, USA) software package. Frequency tables were analyzed using the Chi-square test (X2). To assess the diagnostic value (sensitivity and specificity) of ultrasound elastography compared with the cytological results, cross-tabulation tests were performed.

"Conventional ultrasound and sonoelastography", both conventional sonography and sonoelastography were performed using i22 Philips US machine and high frequency (5-12 MHz) linear probe. All examinations were performed by the same investigator with ten years of experience in thyroid ultrasound. All selected thyroid nodules were assessed by conventional gray-scale and color-Doppler ultrasound. The echogenicity of the nodules was classified into four categories: marked hypoechogenicity, hypoechogenicity, isoechogenicity, and hyperechogenicity. The marked hypoechogenicity was defined as low echogenicity compared with the surrounding strap muscles. The hypoechogenicity, isoechogenicity, and hyperechogenicity were defined according to the comparative echogenicity between the thyroid parenchyma and the nodule. Shape was assessed as the ratio of anteroposterior (A) to transverse ( $\mathrm{T})$ dimensions $(\mathrm{A} / \mathrm{T} \geq 1$ or $<1)$. The margin of the nodule was described as (1) well-circumscribed when the boundary of the nodule was well-defined or the contour of the nodule was smooth and rounded; or (2) poorly-circumscribed when the boundary of the nodule was ill-defined or the contour of the nodule was irregular with jagged edges. Calcification within the nodule was classified into four categories: no calcification, microcalcification, large and dense calcification, and rim calcification. Microcalcification was defined as hyperechoic spots less than $2 \mathrm{~mm}$ with or without acoustic shadowing. The component of the nodule was classified as solid (solid portion $>90 \%$ ), predominantly solid (solid portion $>50 \%$ ), predominantly cystic (cystic portion>50\%), and cystic (cystic portion $>90 \%$ ). The presence and the pattern of blood flow evaluated by color-Doppler imaging were classified as follows: no vascularity-defined as no colorDoppler flow in the periphery or within the nodule; peripheral vascularity-defined as flow in the peripheral position and absent or slight flow in the central part of the nodule; marked intranodular vascularity-defined as more flow in the central part of the nodule than at the periphery. (24). 
"Sonoelastography", was performed after the conventional sonographic examination by the same investigator. With the use of sonoelastographic mode, the probe was placed on the neck with light pressure, and an elastographic region of interest (ROI) was positioned by the operator that included the nodule and sufficient surrounding thyroid tissue to be evaluated. To keep the strain distribution uniform, the probe was pressed to the area with a frequency of 2 to 3 times per second during the cycle of compressing-decompressing in elastography. The level of the pressure was indicated by a 5-point scale meter, which was displayed in real time on the screen. A scale of 2 to 4 was indicative of correct compression. The real-time elastogram was displayed over the gray-scale imaging in a color-coded map: highly elastic tissues (soft) appear in red, less elastic tissues (hard) appear in blue, and intermediate degrees of elastic tissues are shown in green. The sonoelastogram was considered to be reliable only when the elastographic image displayed over the B-mode continued for at least $5 \mathrm{~s}$ with the illuminated indicator showing a value between 2 and 4. In our study, the characteristics of thyroid nodules on sonoelastogram were categorized into 5 patterns (i.e., elasticity scores 1-5). Fig. (1).

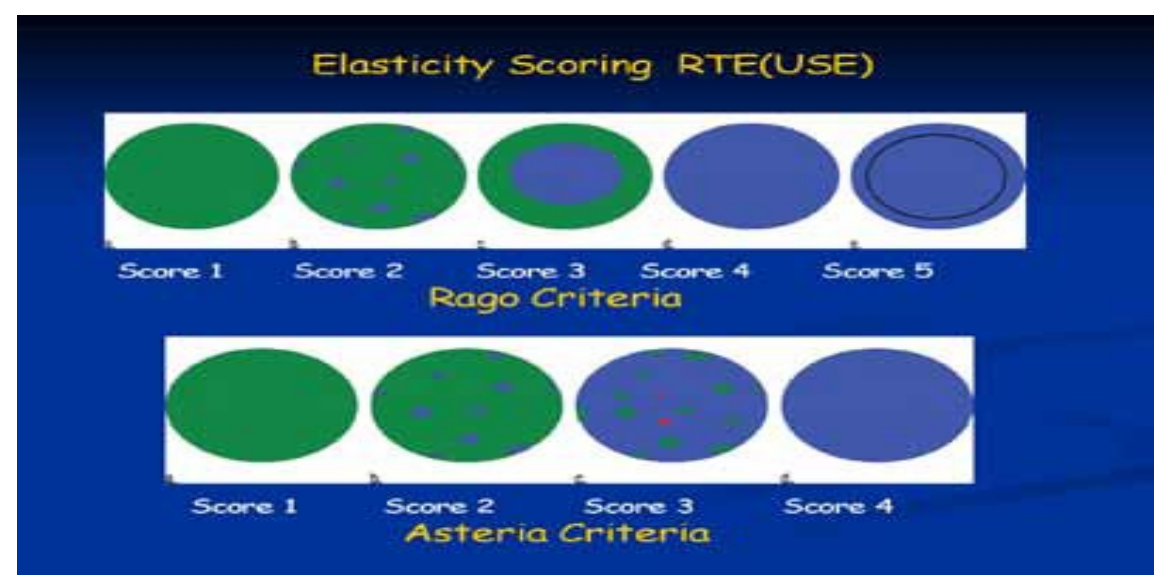

Fig (1) shows elasticity scoring RTE (USE) (25-26).

III. "Results",

Two-hundred and seventy patients with 300 thyroid nodules were successfully evaluated by gray-scale, color-Doppler ultrasound, and 100 patients by sonoelastography. All thyroid nodules were confirmed FNAC by means of surgery. There were 191 benign $(71 \%)$ and 79 malignant $(29 \%)$ nodules. In the 81patients having multiple thyroid lesions, 28 patients had multiple malignant nodules, 60 had multiple benign nodules, and 22 had both multiple benign and malignant nodules. The diagnoses of malignancy included papillary carcinoma $(n=61)$, follicular carcinoma $(n=10)$, anaplastic carcinoma $(n=2)$, and medullary carcinoma $(n=6)$. The diagnoses of benign nodules included nodular goiter $(n=174)$, thyroiditis $(n=8)$, sub acute thyroiditis $(n=4)$, follicular adenoma $(n=3)$, and atypical adenoma $(n=2)$. The mean maximal diameter of the malignant nodules $((1.4 \pm 0.9)$ $\mathrm{cm}$, range from 0.5 to $5 \mathrm{~cm})$ was significantly smaller than that of the benign nodules $((1.7 \pm 1.2) \mathrm{cm}$, range from .5 to $5.4 \mathrm{~cm})(P=0.002)$.

"Ultrasound features of nodules vs. nodule size",To evaluate the relationship between nodule size and ultrasound features, a cutoff of $1 \mathrm{~cm}$ was set as a criterion for dividing small and large nodules. Based on the pathologic findings, there were 111 small nodules (SNs) (malignant 50, benign 61) and 159 large nodules (LNs) (malignant 59, benign 100). The findings of ultrasound features in malignant and benign nodules within SN and LN groups are shown in Table 1 .

Table (1) Frequency of suspicious ultrasound features in malignant and benign nodules according to nodular

\begin{tabular}{|c|c|c|c|c|}
\hline Characteristics & \multicolumn{2}{|c|}{$\mathrm{M}$} & \multicolumn{2}{|c|}{$\mathrm{B}$} \\
\hline & $d 5-10 \mathrm{~mm}(n$ & $10 \mathrm{~mm}(n=61)$ & $d 5-10 \mathrm{~m}$ & $(n=100)$ \\
\hline & $M=50$ & $B=61$ & $M=59$ & $B=100$ \\
\hline $\begin{array}{l}\text { Poorly-circumscribed } \\
\text { margin }\end{array}$ & $45(90 \%)$ & $21(34 \%)$ & $51(86)$ & $24(24 \%)$ \\
\hline Microcalcification & $23(46 \%)$ & $16(26 \%)$ & $41(69 \%)$ & $10(10 \%)$ \\
\hline $\mathrm{A} / \mathrm{T} \geq 1$ & $27(54 \%)$ & $18(30 \%)$ & $5(8 \%)$ & $3(3 \%)$ \\
\hline Marked intranodular & $25(50 \%)$ & $4(7 \%)$ & $25(42 \%)$ & $30(30)$ \\
\hline
\end{tabular}


Roles of gray-scale, Color-Doppler ultrasound and sonoelastography for assessment of thyroid

\begin{tabular}{|c|c|c|c|c|}
\hline vascularity & & & & \\
\hline Elasticity score of 4-6 & $10(20 \%)$ & $3(5 \%)$ & $50(85 \%)$ & $23(23 \%)$ \\
\hline
\end{tabular}

$n$ : number, M: malignant; $B$ : benign; $d$ : diameter.

"Diagnostic accuracy of ultrasound features for malignant nodules", The diagnostic accuracy of the ultrasound features was evaluated separately for SNs and LNs (Table 2) for both SNs and LNs, the presence of marked hypoechogenicity, micro calcification, $\mathrm{A} / \mathrm{T} \geq 1$ ratio, and marked intranodular vascularity had a specificity of $77 \%-97 \%$ and a sensitivity of $8.5 \%-86 \%$. In contrast, the elasticity score of $4-5$ and poorlycircumscribed margin had a specificity of $71 \%-80 \%$ and a sensitivity of $89 \%-93 \%$.

Table (2). Diagnostic accuracy of ultrasound findings for malignant nodules according to nodular size.

\begin{tabular}{|c|c|c|c|c|c|c|c|}
\hline $\mathrm{d} \geq 10 \mathrm{~mm}: \mathrm{n}=111 \mathrm{~ns}$ & $\begin{array}{l}\text { Malignant } \\
(\mathrm{n}=50)\end{array}$ & $\begin{array}{l}\text { Benign, } \\
(\mathrm{n}=61)\end{array}$ & Sensitivity $\%$ & $\begin{array}{c}\text { Specificity } \\
\%\end{array}$ & $\begin{array}{c}\text { PPV } \\
\%\end{array}$ & NPV \% & $\mathrm{P}$ \\
\hline $\begin{array}{l}\text { Poorly-circumscribed } \\
\text { margin }\end{array}$ & 45 & 21 & 90 & 66 & 68 & 34 & $<0.01$ \\
\hline Hypoechogenicity & 40 & 5 & 80 & 91 & 89 & 8 & $<0.01$ \\
\hline microcalcifications & 23 & 16 & 46 & 73 & 58 & 26 & $<0.01$ \\
\hline Intranodular blood flow & 25 & 4 & 50 & 93 & 85 & 6.6 & 0.011 \\
\hline $\mathrm{A} / \mathrm{T} \geq 1 \mathrm{~cm}$ & 27 & 18 & 54 & 70 & 60 & 29.5 & $<0.01$ \\
\hline Elasticity score of $4-5$ & 10 & 4 & 20 & 93 & 71 & 28 & 0.000 \\
\hline \multicolumn{8}{|l|}{$\mathrm{d} \leq 10 \mathrm{~mm}: \mathrm{n}=159 \mathrm{~ns}$} \\
\hline $\begin{array}{l}\text { Poorly-circumscribed } \\
\text { margin }\end{array}$ & 51 & 24 & 86 & 76 & 68 & 32 & $<0.01$ \\
\hline Hypoechogenicity & 17 & 8 & 29 & 92 & 68 & 32 & $<0.01$ \\
\hline microcalcifications & 41 & 10 & 69 & 90 & 80 & 20 & $<<0.01$ \\
\hline Intranodular blood flow & 5 & 3 & 8.5 & 97 & 62 & 37.5 & $<0.01$ \\
\hline $\mathrm{A} / \mathrm{T} \geq 1$ & 25 & 30 & 42.4 & 70 & 45.5 & 54.5 & $<0.01$ \\
\hline Elasticity score of $4-5$ & 50 & 23 & 84 & 77 & 68.5 & 31.5 & 0.001 \\
\hline
\end{tabular}



Fig. (2) Real time ultrasound for 44 yrs old female with papillary carcinoma confirmed by FNA cytology.



Fig. (3) Real time ultrasound for 48 yrs old male with medullary carcinoma confirmed by FNA cytology. 


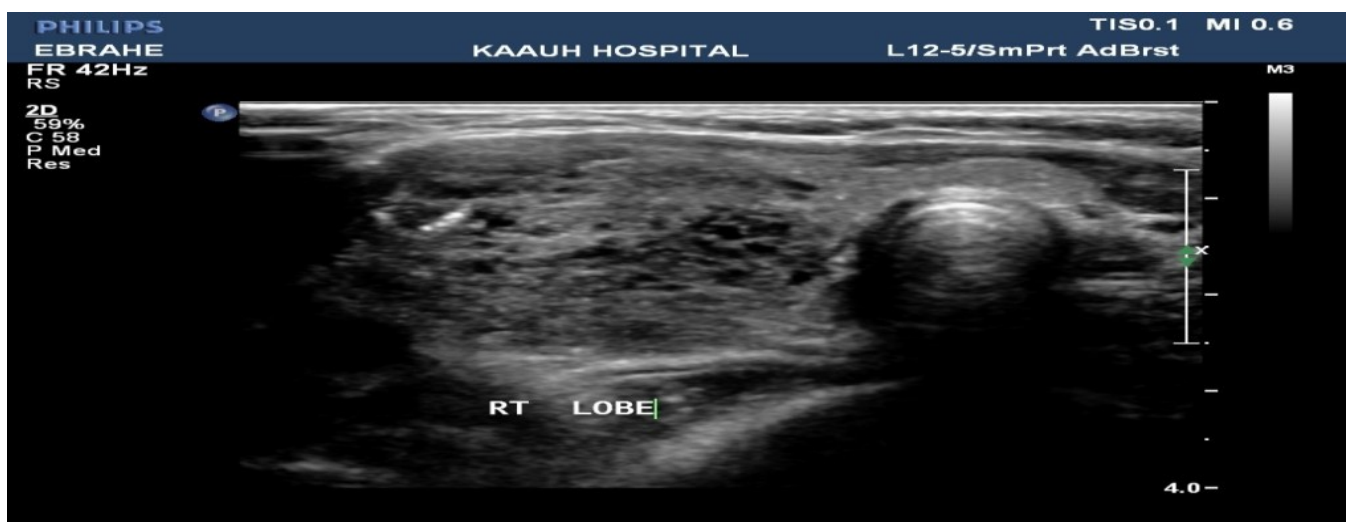

Fig. (4) Conventional ultrasound for 19 yrs old male, with medullary carcinoma confirmed by FNA cytology.

\section{Discussion}

The aims of our prospective study were to the evaluate the predictive value of roles of gray-scale, color-Doppler ultrasound, and sonoelastography for the assessment of thyroid nodule to determine whether nodule size affects the differential diagnosis of benign and malignant, There is a general agreement that ultrasound features indicating a high risk for malignancy should be an indication for an FNAB. Ultrasound features predictive of malignant nodules include the presence of irregular margins, marked hypoechogenicity, microcalcifications, taller-than-wide shape, intranodularvascularity(17,20,21,22,23,24). Ultrasound evaluation of the border of a thyroid nodule can be classified as well-defined or ill-defined. An ill-defined and irregular or microlobulated margin is suggestive of malignancy, but the sensitivity $(48.3 \%-84.4 \%)$ and specificity $(81 \%-$ $97.6 \%)$ are variable. Some investigators have suggested an ill-defined margin as the criterion for the discrimination of malignant from benign nodules, others have suggested an irregular or microlobulated margin as the criterion. $(20,17,23,21,23)$ Poor interobserver agreement when assessing margins may account for these discordant findings. An irregular and microlobulated contour is thought to be the hallmark of thyroid papillary cancers as the neoplastic thyroid follicular epithelia undergo disorganized growth, resulting in an irregular or lobulated appearance. An ill-defined margin may be associated with minimal marginal tumor infiltration of malignancy. Both characteristics suggest malignant infiltration of adjacent thyroid parenchyma with no pseudocapsule formation $(17,19$, and 20). In our study, a poorly-circumscribed, ill-defined or irregular and microlobulated margin (Figs. (Figs.5 $\underline{5}$ and and6), $\underline{6}$ ), was found in $80 \%$ of malignant nodules and $34 \%$ of benign nodules. The sensitivity increased to $90 \%$ in SNs and $86 \%$ in LNs, the specificity was $66 \%$ in SNs and $76 \%$ in LNs, and accuracy exceeded $80 \%$. The frequency of this characteristic had no significant difference between malignant LNs and SNs, but it was less frequent in benign LNs than in benign SNs. The hypoechogenicity of thyroid nodules is not a reliable sign of malignancy since the specificity and PPV are low (17, 18, and 21). Some studies have observed that marked hypoechogenicity was highly specific for diagnosing malignant nodules, but with low sensitivity. In this study, our findings are in accordance with previous studies. In addition, we also find that the predictive ability of marked hypoechogenicity was not relevant to nodular size. Micro calcification is one of the most specific features of thyroid malignancy with a specificity of $71.0 \%-98.8(19,20,21$, and 23$)$. In this study; microcalcifications were more commonly observed in malignant LNs than in malignant SNs. This result was similar to that study $(19,22)$. In our study no significant difference was observed between benign LNs and SNs. Micro calcifications had a specificity of $73 \%$ and a sensitivity of $46 \%$ in SNs. In the LN group, the specificity increased to $90 \%$, and the sensitivity increased to $69 \%$. Therefore, microcalcifications may be more predictive of malignancy in LNs than in SNs. The ratio of the nodular shape $\mathrm{A} / \mathrm{T} \geq 1$ is a reportedly characteristic and very specific feature of malignant thyroid nodules $(17,20,21$, and 23). This appearance may be due to the fact that malignant nodules grow across normal tissue planes, while benign nodules grow parallel to normal tissue planes (20). In our study, we found that this feature was more sensitive in SNs than in LNs. Nearly $54 \%$ of malignant nodules. There are many reports that have evaluated the diagnostic performance of Doppler ultrasound in the assessment of thyroid tumors. Some authors claimed that intranodular vascularity is useful for differentiating benign from malignant thyroid nodules(19) while others disagreed (23).In our study, we defined marked intranodular vascularity as more flow in the central part of the nodule than at the periphery which resulted in a high specificity but a very low sensitivity. We found that the diagnostic accuracy was dependent on tumor size, in which marked intranodular vascularity was more commonly observed in LNs than in SNs in both malignant and benign nodules.Recent studies demonstrated that ultrasound-based elastography could improve detection of malignant thyroid nodules with a high sensitivity and good specificity (20, 19, and 23 ). In our study, the sensitivity of sonoelastography was $84 \%$ and specificity 1 s $77 \%$ in LNs, which could be useful in determining which nodules could be safely observed without biopsy. 


\section{Conclusion:}

The predictive values of microcalcifications, nodular margins, $\mathrm{A} / \mathrm{T}$ ratio, and marked intranodular vascularity depend on nodule size, but the predictive values of echogenicity and elastography do not depend on nodule size.

\section{Acknowledgment:}

This project was funded by the Deanship of Scientific Research (D S R), King Abdulaziz University, Jeddah, under grant no. (371/142/1432). The authors, therefore, acknowledge with Thanks DSR technical and financial support.

\section{References}

[1]. Tumbridge WM, Evered DC, Hall R, et al. The spectrum of thyroid disease in a community: the Whick-ham Survey. Clin Endocrinol (Oxf) 1997;7:481-93

[2]. Hong Y, Liu X, Li Z, Zhang X, Chen M, and Luo Z. Real-time Ultrasound Elastography in the Differential Diagnosis of Benign and Malignant Thyroid Nodules. JUM July 1, 2009 vol. 28no. 7 861-867

[3]. Kim EK, Park CS, Chung WY, et al. New sonographic criteria for recommending fine-needle aspiration biopsy of nonpalpable solid nodules of the thyroid. AJR Am J Roentgenol 2002;178:687-91

[4]. Park JY, Lee HJ, Jang HW, et al. A proposal for a thyroid imaging reporting and data system for ultrasound features of thyroid carcinoma. Thyroid2009;19:1257-64

[5]. Sipos JA. Advances in ultrasound for the diagnosis and management of thyroid cancer. Thyroid 2009;19:1363-72

[6]. Hong YJ, Son EJ, Kim EK, et al. Positive predictive values of sonographic features of solid thyroid nodule. Cli Imaging 2010;34:127-33

[7]. Moon HJ, Kwak JY, Kim MJ, et al. Can vascularity at power Doppler US help predict thyroid malignancy? Radiology 2010;255:260-69

[8]. Choi YJ, Yun JS, Kim DH. Clinical and ultrasound features of cytology diagnosed follicular neoplasm. Endocr J 2009;56:383-89

[9]. Hagag P, Strauss S, Weiss M. Role of ultrasound-guided fine-needle aspiration biopsy in evaluation of nonpalpable thyroid nodules. Thyroid1998;8:989-95

[10]. Luo S, Kim EH, Dighe M, Kim Y. Screening of thyroid nodules by ultrasound elastography using diastolic strain variation. Conf Proc IEEE Eng Med Biol Soc. 2009;2009:4420-3. doi: 10.1109/IEMBS.2009.5332744.

[11]. Albert G. Burger. Fine-Needle Aspiration Biopsy with BRAF Analysis and Elastography are Slightly More Efficient in Diagnosing Papillary Thyroid Cancers than FNAB and Thyroid Ultrasound. Clin Thyroidol 2012;24:6-7.

[12]. S. Merinoa, J. Arrazolaa, A. Cárdenasa, M. Mendozab, P. De Miguelc, C. Fernándezd and T. Ganadoa. Utility and Interobserver Agreement of Ultrasound Elastography in the Detection of Malignant Thyroid Nodules in Clinical Care AJNR 2011 32: $2142-2148$

[13]. Frey H. Realtime elastography: a new ultrasound procedure for the reconstruction of tissue elasticity [in German]. Radiologe 2003;43:850-55

[14]. Asteria C, Giovanardi A, Pizzocaro A, Cozzaglio L, Morabito A, Somalvico F, Zoppo A.US-elastography in the differential diagnosis of benign and malignant thyroid nodules. 2008 May;18(5):523-31.

[15]. Itoh A, Ueno E, Tohno E, Kamma H, Takahashi H, Shiina T, Yamakawa M, Matsumura T .Breast disease: clinical application of US elastography for diagnosis. Radiology, 2006; 239:341-350.

[16]. Kakkos SK, Scopa CD, Chalmoukis AK, Karachalios DA, Spiliotis JD, Harkoftakis JG, Karavias DD,

[17]. Androulakis JA, Vagenakis AG. Relative risk of cancer in sonographically detected thyroid nodules with calcifications. J Clin Ultrasound. 2000;28(7):347-352. doi: 10.1002/1097-0096(200009)28:7<347::AID-JCU5>3.3.CO;2-F. [ubMed] [Cross Ref]

[18]. Kim EK, Park CS, Chung WY, Oh KK, Kim DI, Lee JT, Yoo HS. New sonographic criteria for recommending fine-needle aspiration biopsy of nonpalpable solid nodules of the thyroid. Am J Roentgenol. 2002;178(3):687-691. [PubMed]

[19]. Wienke JR, Chong WK, Fielding JR, Zou KH, Mittelstaedt CA. Sonographic features of benign thyroid nodules: interobserver reliability and overlap with malignancy. J Ultrasound Med. 2003;22(10):1027-1031. [PubMed].

[20]. Reading CC, Charboneau JW, Hay ID, Sebo TJ. Sonography of thyroid nodules: a "classic pattern" diagnostic approach. Ultrasound Q. 2005;21(3):157-165. doi: 10.1097/01.ruq.0000174750.27010.68. [PubMed] [Cross Ref]

[21]. Hoang JK, Lee WK, Lee M, Johnson D, Farrell S. US features of thyroid malignancy: pearls and pitfalls. Radiographics. 2007;27(3):847-860. doi: 10.1148/rg.273065038. [PubMed] [Cross Ref]

[22]. Moon WJ, Jung SL, Lee JH, Na DG, Baek JH, Lee YH, Kim J, Kim HS, Byun JS, Lee DH, et al. Benign and malignant thyroid nodules: US differentiation-multicenter retrospective study. Radiology. 2008;247(3):762-770. doi: 10.1148/radiol.2473070944. [PubMed] [Cross Ref].

[23]. Rubaltelli L, Stramare R, Tregnahi A, Scagliori E, Cecchelero E, Mannucci M, Gallinaro E, Beltrame V. The role of sonoelastography in the differential diagnosis of neck nodules. J Ultrasound. 2009;12(3):93-100. doi: 10.1016/j.jus.2009.05.001. [PMC free article] [PubMed] [Cross Ref]

[24]. Bojunga J, Herrmann E, Meyer G, Weber S, Zeuzem S, Friedrich-Rust M. Real-time elastography for the differentiation of benign and malignant thyroid nodules: a meta-analysis. Thyroid. 2010;20(10):1145-1150. doi: 10.1089/thy.2010.0079. [PubMed] [Cross Ref].

[25]. 16 Yan CX, Luo ZY, Liu XM, Huang PT, Mo GQ, Hong YR, Wen Q, Pan MQ, Weng HF

[26]. Zhonghua Yi Xue Za Zhi. 2013 Jun 4; 93(21):1630-3. Chinese.

[27]. Rago T, Santini F, Scutari M, Pinchera A, Vitti P Elastography: new developments in ultrasound for predicting malignancy in thyroid nodules. J Clin Endocrinol Metab 2007, 92;2917-2922.

[28]. Asteria C, Giovanardi A, Pizzocaro A, Cozzaglio L, Morabito A, Somalvico F, Zoppo A . US-elastography in the differential diagnosis of benign and malignant thyroid nodules. Thyroid, 2008; 18:523-531. 